\title{
Craniotomy Complicated by Severe Metabolic Acidosis Requiring Massive Transfusion in an Infant on Ketogenic Diet Therapy for Intractable Epilepsy
}

\author{
Seth B Hayes ${ }^{1,2}$, Dolly M Munlemvo (D)', Holly C Gillis ${ }^{1,2}$, Joseph D Tobias $\mathbb{D}^{1,2}$ \\ 'Department of Anesthesiology \& Pain Medicine, Nationwide Children's Hospital, Columbus, OH, USA; ${ }^{2}$ Department of Anesthesiology \& Pain \\ Medicine, The Ohio State University College of Medicine, Columbus, OH, USA
}

Correspondence: Seth B Hayes, Department of Anesthesiology \& Pain Medicine, Nationwide Children's Hospital, 700 Children's Drive, Columbus, $\mathrm{OH}, 43205$, USA, Tel +I 6I4 722-4200, Fax +I 614 722-4203, Email Seth.Hayes@nationwidechildrens.org

\begin{abstract}
The induction of a ketotic state through dietary manipulation, known as the ketogenic diet (KD), is an alternative or supplementary treatment to drug-resistant epilepsy. By sustaining a ketogenic state, the KD results in various biological adaptations which contribute to its success as an anti-seizure therapy. While the induction and maintenance of ketosis generally results in only a low-grade metabolic acidosis, various exogenous stresses such as surgery and anesthetic care may disrupt homeostasis resulting in exaggerated ketosis and severe metabolic acidosis. Metabolic acidosis may have significant effects on various physiologic functions including cardiovascular performance, coagulation function, and electrolyte balance. We present a 7-month-old patient receiving a KD who presented for craniotomy and resection of an epileptogenic focus. During intraoperative care, progressive acidosis and hyperchloremia were noted with ongoing tissue fragility and hyperemia, parenchymal friability, and coagulopathy. Though the acidosis was temporarily blunted by administration of sodium bicarbonate and a change to sodium acetate containing fluids, ultimately poor hemostasis resulted requiring significant blood product transfusion. The metabolic effects of the KD are reviewed with emphasis on acid-base disturbances and impact on coagulation function.
\end{abstract}

Keywords: ketogenic diet, seizures, metabolic acidosis, ketosis, coagulopathy

\section{Introduction}

The ketogenic diet $(\mathrm{KD})$ is an alternative or supplementary treatment for drug-resistant epilepsy. ${ }^{1}$ The high fat, low carbohydrate intake of the KD induces a state of ketosis which is postulated to have anticonvulsant effects through mechanisms such as enhanced neuronal energy reserves, antioxidant activity, and anti-inflammatory actions, among others. ${ }^{1-3}$ During the perioperative period, these biochemical changes may pose potential risks such as metabolic acidosis, dehydration, and altered serum electrolytes due to increased urinary losses of potassium, magnesium, and calcium. The acidosis established by the KD is generally a mild or low-grade metabolic acidosis with limited impact on the patient's acidbase status. ${ }^{2}$ Despite the increase in serum ketones, the changes in serum $\mathrm{pH}$ and bicarbonate levels are limited in the majority of patients adhering to the $\mathrm{KD} .{ }^{4}$ In these patients, serum $\beta$-hydroxybutyrate levels vary from $4-6 \mathrm{mmol} / \mathrm{L}$ (normal $\leq 1.5 \mathrm{mmol} /$ $\mathrm{L}$ ), indicating a mild degree of ketosis. ${ }^{3,5}$ The extent of the ketosis and the impact on $\mathrm{pH}$ is significantly less than what occurs in pathologic processes such as diabetic ketoacidosis. ${ }^{3}$ However, significant metabolic acidosis may occur with prolonged nil per os (NPO) times, during prolonged surgical procedures, or from other stresses imposed on the acid-base system including dilutional acidosis from the administration of non-buffer containing intravenous fluids or the concomitant administration of anti-seizure medications that inhibit carbonic anhydrase (topiramate or zonisamide). We present the case of a 7-month-old patient receiving a KD who presented for craniotomy and resection of an epileptogenic focus. During intraoperative care, 
progressive acidosis and hyperchloremia was noted with ongoing tissue fragility and hyperemia, parenchymal friability, poor hemostasis, and coagulopathy, requiring significant blood product transfusion. The metabolic effects of the KD are reviewed with emphasis on acid-base disturbances and impact on coagulation function.

\section{Case Report}

Review of this case and presentation in this format followed the guidelines of the Institutional Review Board (IRB) of Nationwide Children's Hospital (Columbus, Ohio). While IRB approval is not required by our institution for case reports, consent was obtained for this publication from the patient's guardian.

A 7-month-old, $9 \mathrm{~kg}$ child with medically intractable epilepsy and intermittent status epilepticus presented for resection of left temporal, parietal, and occipital epileptogenic regions. The child was born full term by uncomplicated vaginal delivery. There was no significant past medical history until infantile spasms and focal seizures with episodes of apnea were diagnosed at 4 weeks of age. Though sporadic at first, over the next 6 months these events progressed to greater than twenty partial and generalized tonic-clonic seizures per day. Despite home medications including five oral anti-seizure agents (zonisamide, lacosamide, vigabatrin, levetiracetam, and phenobarbital), the patient's increasingly frequent clinical and subclinical seizures required multiple hospital admissions. The source of this patient's epilepsy is thought to be multifactorial. Dysmorphic neurons in the setting of diffuse cortical dysplasia were found on pathology. Additionally, the patient has a history of cytomegalovirus encephalitis from congenital infection which may have contributed to the clinical presentation.

In an attempt to reduce the seizure burden, ketogenic diet therapy was initiated approximately two weeks prior to surgery and achieved ketosis. Preoperative assessment included long term electroencephalography (EEG) monitoring and brain imaging using MRI, PET, and CT imaging with stereotactic grid characterization. Pertinent preoperative laboratory tests included hematocrit $32.9 \%$, serum bicarbonate $22 \mathrm{mmol} / \mathrm{L}$, glucose of $56 \mathrm{mg} / \mathrm{dL}$, positive urinary ketones $(5 \mathrm{mg} / \mathrm{dL})$, and elevated $\beta$-hydroxybutyric acid at $5.69 \mathrm{mmol} / \mathrm{L}$. The patient's electrolyte, blood count, and coagulation panels were otherwise within normal limits. The ketogenic diet was maintained preoperatively by nasogastric tube feeding of Ketovie ${ }^{\circledR} 3: 1$ and $\mathrm{NanoVM}^{\mathbb{B}}$ ketogenic formulas until midnight prior to the surgical procedure. After that, the patient was kept nil per os. Once in the operating room, standard American Society of Anesthesiologists' monitors were placed. Anesthesia was induced by the inhalation of sevoflurane in nitrous oxide and oxygen. Following anesthetic induction, a peripheral intravenous cannula was placed, and endotracheal intubation was facilitated by the administration of rocuronium. Following endotracheal intubation, a second peripheral intravenous cannula and an arterial cannula were placed. The operating room table was turned $180^{\circ}$ in preparation for the procedure. Maintenance anesthesia included inhaled sevoflurane (2-4\%) in air/oxygen and a remifentanil infusion titrated to clinical need. Intermittent doses of rocuronium were used to provide ongoing neuromuscular blockade. In place of the inhaled sevoflurane, a propofol infusion was used briefly during a period of intraoperative electrocorticography. Other medications administered during this case included midazolam, fentanyl, cefazolin, phenylephrine, dexamethasone, and mannitol. Throughout the procedure, glucose-containing medications as well as medications known to precipitate gluconeogenesis were identified and avoided or their use discussed with the neurology and neurosurgery teams. ${ }^{2}$

During the initial part of the surgical procedure, intravenous fluids included $0.9 \%$ normal saline with no added glucose. Laboratory parameters obtained during the procedure are listed in Table 1. An initial arterial blood gas (ABG) revealed a mild metabolic acidosis ( $\mathrm{pH} 7.33$ ) with a base deficit of $-7.7 \mathrm{mmol} / \mathrm{L}$. Following fluid resuscitation with $0.9 \%$ normal saline of $700 \mathrm{~mL}$ ( $70 \mathrm{~mL} / \mathrm{kg}$ during the first 7 hours of the surgical procedure), the metabolic acidosis continued to worsen (nadir $\mathrm{pH}$ 7.06). Three doses of $8.4 \%$ sodium bicarbonate $\left(\mathrm{NaHCO}_{3}\right)$, each of $1 \mathrm{mEq} / \mathrm{kg}$, were administered with a moderate correction of the metabolic acidosis and an increase in the $\mathrm{pH}$ to 7.27. However, the administration of $\mathrm{NaHCO}_{3}$ was limited by an increase in the serum sodium to $154 \mathrm{mEq} / \mathrm{L}$. A venous chemistry panel and serum lactate were sent to allow a further evaluation of the ongoing metabolic acidosis (Table 2). Although the serum lactate was within normal limits, the presence of hyperchloremia $(116 \mathrm{mEq} / \mathrm{L})$ prompted a switch to a $0.9 \%$ sodium acetate solution for maintenance, ongoing resuscitation, and replacement of 3rd space losses. With the development of metabolic acidosis, the surgeons noted parenchymal hyperemia, tissue friability, and poor hemostasis. A coagulation panel revealed an activated partial thromboplastin time (aPTT) of 29 seconds (normal 24-36 seconds), a prothrombin time of 17.9 seconds (normal 12.4-14.7 seconds), international normalized ratio of 1.5, and fibrinogen of $113 \mathrm{mg} / \mathrm{dL}$ (normal 170- 
Table I Laboratory Results and Fluid Management

\begin{tabular}{|c|c|c|c|c|c|c|c|c|c|c|c|c|c|c|c|c|}
\hline Parameter and Time & 1022 & 1144 & 1303 & 1414 & 1428 & 1522 & 1529 & 1546 & 1607 & 1640 & 1641 & 1739 & 1819 & 1854 & 1904 & 1918 \\
\hline $\mathrm{pH}$ & 7.33 & 7.19 & 7.20 & 7.08 & & 7.06 & \multirow{12}{*}{$\begin{array}{l}\text { Chemistry results obtained (See } \\
\text { Table 2) }\end{array}$} & & 7.27 & 7.20 & & 7.16 & 7.08 & 7.29 & 7.16 & 7.16 \\
\hline $\mathrm{PaCO} 2(\mathrm{mmHg})$ & 33 & 42 & 32 & 35 & & 36 & & & 37 & 32 & & 36 & 44 & 34 & 48 & 49 \\
\hline $\mathrm{PaO} 2(\mathrm{mmHg})$ & 136 & 158 & 115 & 157 & & 178 & & & 201 & 60 & & 227 & 117 & 124 & 149 & 125 \\
\hline Oxygen saturation (\%) & 100 & 100 & 98 & 99 & & 99 & & & 100 & 91 & & 100 & 98 & 100 & 100 & 99 \\
\hline Base deficit (mmol/L) & 7.7 & 11.4 & 14.5 & 18.2 & & 19.0 & & & 9.4 & 14.6 & & 14.8 & 15.6 & 9.5 & 10.9 & 11.3 \\
\hline Glucose (mg/dL) & 54 & 82 & 57 & 108 & & 114 & & & 108 & 106 & & 139 & 139 & 139 & 231 & 237 \\
\hline Hematocrit (\%) & 30.5 & 28.5 & 22.4 & 26.1 & & 31.8 & & & 22.9 & 31.0 & & 24.3 & 24.1 & 16.6 & 30.1 & 38.3 \\
\hline Hemoglobin (gm/dL) & 10.0 & 9.3 & 7.3 & 8.5 & & 10.4 & & & 7.5 & 10.2 & & 7.9 & 7.9 & 5.4 & 9.8 & 12.5 \\
\hline lonized calcium (mmol/L) & 1.08 & 1.10 & 1.13 & 1.10 & & 1.14 & & & 0.93 & 0.77 & & 1.11 & 1.05 & 0.94 & 0.63 & 0.97 \\
\hline Sodium (mEq/L) & 144 & 144 & 142 & 146 & & 144 & & & 154 & 153 & & $15 \mid$ & 155 & 159 & 155 & 152 \\
\hline Potassium (mEq/L) & 4.1 & 3.4 & 3.5 & 3.3 & & 4.4 & & & 3.3 & 4.6 & & 3.7 & 3.6 & 3.3 & 3.6 & 5.0 \\
\hline Lactate $(\mathrm{mmol} / \mathrm{L})$ & & & & & 0.5 & & & 0.8 & & & 1.1 & & & & & \\
\hline \multicolumn{17}{|l|}{ Fluids administered } \\
\hline $0.9 \%$ Sodium chloride & \multicolumn{8}{|c|}{$\mathrm{I} \rightarrow \rightarrow \rightarrow \rightarrow \rightarrow \rightarrow \rightarrow \rightarrow \rightarrow \rightarrow \rightarrow \rightarrow \rightarrow \rightarrow 700 \mathrm{~mL}$} & & & & & & & & \\
\hline $8.4 \%$ Sodium bicarbonate & & & & & & & $30 \mathrm{mEq}$ & & & & & & & & & \\
\hline $0.9 \%$ Sodium acetate & & & & & & & & & \multicolumn{4}{|c|}{$35 \mathrm{~mL} / \mathrm{hr} \rightarrow \rightarrow \rightarrow \rightarrow \rightarrow$} & & & & \\
\hline 10\% Dextrose & & & & & & & & & & & & $10 \mathrm{~mL}$ & & & & \\
\hline $5 \%$ Dextrose $-0.45 \%$ NS & & & & & & & & & & & & & \multicolumn{4}{|c|}{$35 \mathrm{~mL} / \mathrm{hr} \rightarrow \rightarrow \rightarrow \rightarrow \rightarrow$} \\
\hline $5 \%$ Albumin & & & & & & $100 \mathrm{~mL}$ & & & & & & $20 \mathrm{~mL}$ & & & & \\
\hline
\end{tabular}


Table 2 General Chemistry Panel

\begin{tabular}{|l|l|l|}
\hline Sodium & 140 & $\mathrm{mEq} / \mathrm{L}$ \\
\hline Potassium & 4.3 & $\mathrm{mEq} / \mathrm{L}$ \\
\hline Chloride & 116 & $\mathrm{mEq} / \mathrm{L}$ \\
\hline Carbon dioxide & 11 & $\mathrm{mEq} / \mathrm{L}$ \\
\hline Blood urea nitrogen & 8 & $\mathrm{mg} / \mathrm{dL}$ \\
\hline Creatinine & 0.25 & $\mathrm{mg} / \mathrm{dL}$ \\
\hline Glucose & 87 & $\mathrm{mg} / \mathrm{dL}$ \\
\hline Calcium & 8.0 & $\mathrm{mg} / \mathrm{dL}$ \\
\hline Magnesium & 2.0 & $\mathrm{mg} / \mathrm{dL}$ \\
\hline Phosphorus & 6.1 & $\mathrm{mg} / \mathrm{dL}$ \\
\hline
\end{tabular}

$410 \mathrm{mg} / \mathrm{dL})$. These abnormalities were not immediately treated as the rotational thromboelastogram $\left(\mathrm{ROTEM}^{\circledR}\right)$ was within normal limits. Due to the ongoing bleeding and tissue friability, the team in the operating room (pediatric anesthesiology, neurology, and neurosurgery) discussed aborting the procedure with the plan to return for completion after patient stabilization. However, as the acidosis had begun to improve and the coagulation profile and ROTEM ${ }^{\circledR}$ were reassuring, the decision was made to continue.

As the surgery progressed, the patient's severe acidosis returned. After discussion with the neurology team, ketosis labs were sent which revealed an elevated $\beta$-hydroxybutyric acid level of $7.3 \mathrm{mmol} / \mathrm{L}$ and blood glucose of $106 \mathrm{mg} / \mathrm{dL}$. At this time, the decision was made to reverse the ketogenic state in order to minimize the contribution of the ketoneinduced metabolic acidosis. A $10 \mathrm{~mL}$ bolus $(1.1 \mathrm{~mL} / \mathrm{kg})$ of $10 \%$ dextrose was administered intravenously, $0.9 \%$ sodium acetate was discontinued, and 5\% dextrose in $0.45 \%$ normal saline maintenance fluids were started at $35 \mathrm{~mL} / \mathrm{hr}$. During these adjustments, the surgical team noted ongoing brain tissue friability. A subsequent ABG assessment noted another decrease of the $\mathrm{pH}$ to 7.08 which was concomitant with oozing from prior resection areas and spontaneous venous bleeding occurring in multiple sites on the surgical field. Coagulopathy was treated by the administration of fresh frozen plasma and cryoprecipitate. Persistent blood loss required the ongoing transfusion of packed red blood cells. Surgical bleeding became more difficult to control despite multiple attempts at surgical hemostasis. A repeat coagulation panel was ordered, but a blood sample was not sent as efforts to stabilize the patient became increasingly demanding. Tranexamic acid (TXA) was administered, and the massive transfusion protocol was activated. The surgical team elected to proceed with rapid closure and packing of the surgical field without replacement of the bone flap. The patient was transported to the pediatric ICU with ongoing endotracheal intubation and mechanical ventilation to allow for further stabilization and resuscitation. Intraoperatively, the patient received $1031 \mathrm{~mL}(115 \mathrm{~mL} / \mathrm{kg})$ of packed red blood cells, $310 \mathrm{~mL}(35 \mathrm{~mL} / \mathrm{kg})$ of fresh frozen plasma, $211 \mathrm{~mL}(24 \mathrm{~mL} / \mathrm{kg})$ of platelets, and $39 \mathrm{~mL}(4-5 \mathrm{~mL} / \mathrm{kg})$ of cryoprecipitate in addition to boluses of 5\% albumin and multiple doses of calcium gluconate. The estimated blood loss was $700 \mathrm{~mL}$.

In the ICU, the patient's acidosis quickly improved ( 7.44 on postoperative day one) with resumption of sodium acetate maintenance fluids. Hemostasis was achieved by surgical packing and correction of the metabolic acidosis. As a result, the initial hemoglobin on arrival to the unit was $19.3 \mathrm{~g} / \mathrm{dL}$. A coagulation panel performed on postoperative day one was entirely within normal limits. As a result of the intraoperative complications, the ketogenic diet was not restarted after the procedure. The patient returned to the operating room one week later for bone flap replacement, hematoma evacuation, and further resection. Unfortunately, good seizure control was still unable to be established, and a left hemispherectomy was subsequently performed. The patient recovered well from this surgery and was transferred out of the pediatric ICU one month after the initial operation. On postoperative day 50, the patient was discharged home with significantly improved seizure control. Since discharge, the patient's parent has reported a few events of questionable 
Table 3 Summary of Case Events and Recommendations for Intraoperative Management of Patients Requiring Ketogenic Diet Undergoing Epilepsy Surgery

\begin{tabular}{|c|c|}
\hline Event & Recommendations \\
\hline $\begin{array}{l}\text { 7-month-old patient with epilepsy on KDT presents } \\
\text { for epilepsy surgery }\end{array}$ & $\begin{array}{l}\text { - Review patient level comorbidities and risk factors } \\
\text { - Obtain baseline metabolic and hematologic parameters, discern pharmacologic and } \\
\text { dietary management } \\
\text { - Complete physical exam } \\
\text { - Devise intraoperative respiratory and fluid management strategies to maintain } \\
\text { normal acid-base status based on history and physical exam } \\
\text { - Notify ICU of pending admission }\end{array}$ \\
\hline Development of intraoperative acidosis & $\begin{array}{l}\text { - Differentiate gap versus non-gap causes of metabolic acidosis vs } \\
\text { respiratory acidosis } \\
\text { - Resuscitate using balanced salt solution }\end{array}$ \\
\hline $\begin{array}{l}\text { Development of coagulopathy and surgical site } \\
\text { bleeding }\end{array}$ & $\begin{array}{l}\text { - Evaluate adequacy of resuscitation to date } \\
\text { - Judicious transfusion parameters given implications for acid-base status }\end{array}$ \\
\hline Cessation of surgical management, transport to ICU & $\begin{array}{l}\text { - Transport to ICU when completion of surgical procedure is not feasible given } \\
\text { metabolic derangements and surgical site hemorrhage }\end{array}$ \\
\hline Return to operating room for completion of surgery & - Repeat preoperative optimization prior to any return to operating room \\
\hline $\begin{array}{l}\text { Discharge to home following optimization of seizure } \\
\text { control }\end{array}$ & $\begin{array}{l}\text { - Clear and frequent intradepartmental communication allows for optimal care of } \\
\text { patients undergoing epilepsy surgery }\end{array}$ \\
\hline
\end{tabular}

seizure activity but reports markedly improved responsiveness and awareness. Although diagnoses of global developmental delay and quadriplegic cerebral palsy with a mixture of spastic, hypotonic, and dystonic tone have since been made, the patient continues to improve through regular participation with physical and occupational therapy. A summary of events and recommendations for intraoperative management of patients requiring ketogenic diet undergoing epilepsy surgery can be found in Table 3 .

\section{Discussion}

The KD results in various biological adaptations which contribute to its success as an anti-seizure therapy. ${ }^{2}$ Though it can serve as a useful adjunct to pharmacologic agents, the diet can be limited by several adverse effects. Gastrointestinal symptoms are most common, including nausea, constipation, and abdominal pain. Hyperlipidemia, kidney stones, pancreatitis, and liver transaminitis can occur. ${ }^{2,6,7}$ Additionally, as a consequence of sustaining a ketogenic state, the elevation of ketone bodies may induce a chronic metabolic acidosis. The acidosis established by the KD has generally been described as low-grade, having only a small effect on acid-base balance. ${ }^{2}$ Despite the increase in serum and urine ketones, the changes in serum $\mathrm{pH}$ and bicarbonate levels are limited in most patients. ${ }^{4}$ Serum $\beta$-hydroxybutyrate levels vary from $4-6 \mathrm{mmol} / \mathrm{L}$ (normal $\leq 1.5 \mathrm{mmol} / \mathrm{L}$ ), indicating a mild degree of ketosis. ${ }^{3,5}$ However, as noted in our patient, significant changes in $\mathrm{pH}$ with metabolic acidosis may occur during prolonged surgical procedures or when there are additional stresses on acid-base balance. The dilutional acidosis from the administration of non-buffer containing intravenous fluids, the administration of blood and blood products, or the concomitant use of anti-seizure agents that inhibit carbonic anhydrase (zonisamide in our patient) may each contribute to clinically substantial acidemia. Valencia et al noted the development of metabolic acidosis in 3 of 9 patients on a KD for the treatment of medically intractable epilepsy during procedures lasting longer than 3 hours. ${ }^{8}$ Intravenous bicarbonate was administered to the 3 patients to correct the acidosis while additional fluid resuscitation was also administered to one patient. The authors concluded that children on the KD do appear to be at risk for developing metabolic acidosis during general anesthesia, particularly during prolonged surgical procedures. They noted no association with the preoperative requirement for oral citrate or the concomitant administration of topiramate and the development of intraoperative acidosis. However, they note that 
authors have reported the occurrence of acidosis with the administration of topiramate and the use of a KD. Takeoka et al found a $>15 \%$ reduction in serum bicarbonate in 11 of 14 patients and $>20 \%$ reduction in 9 of 14 patients in whom cotreatment of topiramate and a KD was used. ${ }^{9}$

Other factors can be responsible for or add to the development of acidosis during intraoperative care. Examples include tissue hypoperfusion and the development of lactic acidosis, dilutional acidosis from the administration of non-bicarbonate containing fluids as was noted in our patient, and the administration of blood and blood products with their associated low $\mathrm{pH} .{ }^{10}$ In our patient, tissue hypoperfusion was ruled out by documentation of a normal intraoperative serum lactate. Dilutional acidosis from resuscitation with non-bicarbonate containing fluids is common during intraoperative care and results not only in a decrease in the $\mathrm{pH}$ but an increase the serum chloride concentration as noted in our patient following the administration of $70 \mathrm{~mL} / \mathrm{kg}$ of $0.9 \%$ normal saline. ${ }^{10}$ Although generally well tolerated, when there are other factors resulting in acidosis, treatment may be required to maintain a $\mathrm{pH} \geq 7.25$. In our patient, the administration of sodium bicarbonate and the change to sodium acetate containing fluids resulted in a blunting of the metabolic acidosis. Further corrective measures ultimately included the decision to reverse the patient's ketosis with dextrose containing fluids.

While generally well tolerated, significant acidosis $(\mathrm{pH} \leq 7.20)$ may result in direct depression of myocardial contractility and systemic vasodilatation resulting in hypotension. Additionally, acidosis may depress the normal response to vasoactive agents. ${ }^{11}$ Other cardiovascular complications including arrhythmias with QT interval prolongation, ST-T wave changes, and even cardiac arrest have all been anecdotally noted as potential adverse effects associated with acidosis. ${ }^{11-13}$ At the tissue and cellular level, acidosis stimulates the pro-inflammatory cascade with the release of tumor necrosis factor and nitric oxide and impairs tissue oxygenation resulting in reduced ATP production. ${ }^{11,13}$

Furthermore, acidosis may affect various steps in the coagulation cascade including the impairment of platelet aggregation, prolongation of thrombin generation, and increasing fibrinogen breakdown. ${ }^{14-16}$ Using an infusion of hydrochloric acid $(\mathrm{HCl})$ to adjust $\mathrm{pH}$ in blood collected from healthy adult volunteers, Engstrom et al reported coagulation impairment at a $\mathrm{pH}$ of 7.15 when compared to a $\mathrm{pH}$ of 7.4. These findings were measurable 90 seconds after $\mathrm{HCl}$ injection as measured by thromboelastography. ${ }^{16}$ The authors noted that the impairment found when lowering $\mathrm{pH}$ from 7.4 to 7.15 was almost identical to the impairment seen when the temperature was lowered from $36^{\circ} \mathrm{C}$ to $32^{\circ} \mathrm{C}$. Concerns have also been raised regarding coagulation function specifically in patients on the $\mathrm{KD}$. Based on their clinical observations and parental reports of increased bruising, Berry-Kravis et al reviewed bruising and coagulation function in a cohort of 51 patients on the KD. ${ }^{17} \mathrm{~A}$ significant increase in bruising or other minor bleeding was reported or observed in 16 of 51 patients (31.4\%). No differences were noted based on gender or the number of anti-seizure medications that the patients were receiving although the group with bruising/bleeding was significantly younger. A more in-depth investigation of coagulation function was performed in 6 patients, 5 of whom had prolonged bleeding times and diminished responsiveness to various platelet aggregating agents with no evidence of a release defect. These abnormalities normalized in the one patient who was retested after stopping the KD and could be corrected by the administration of desmopressin (DDAVP ${ }^{\circledR}$ ). These authors postulated that the coagulation defect may be related to a pre-existing susceptibility and/or a diet-induced depression of platelet responsiveness related to changes in platelet membrane lipid composition and/or concentration with a resultant effect on the function of membrane-embedded proteins. However, Dressler et al noted no coagulation abnormalities in a consecutive series of 162 children receiving the KD. ${ }^{18}$ Serial measurements of platelet counts and global coagulation tests (aPTT, PT, and fibrinogen) were obtained at baseline and during KD therapy (at 1, 3, 6 and 12 months). The authors noted no clinical bleeding either during routine activities of daily life or during surgery and no clinically significant abnormalities in coagulation function; however, platelet function was not assessed.

These concerns are magnified in patients undergoing procedures that are associated with significant surgical bleeding including craniotomy and neurosurgical procedures. ${ }^{19}$ During such procedures, the rapid administration of large volumes of blood products may result in secondary dilutional coagulation due to significant blood loss in excess of 1-2 blood volumes. ${ }^{20}$ In our patient, a combination of factors was likely responsible for the intraoperative bleeding that we noted including the surgical procedure, the large volume of blood products transfused, acidosis, and the KD. 


\section{Conclusion}

The perioperative anesthetic management of patients undergoing surgery for intractable epilepsy remains challenging. The preoperative assessment of patients receiving a KD should include a metabolic evaluation including serum electrolytes, acid-base status, and glucose. Intraoperative fluid therapy including buffer containing fluids may mitigate the acidosis from the KD and associated anti-seizure agents. Patients on the diet undergoing anticoagulation or surgery should be evaluated for clinical symptoms of bleeding. If there is a clinical history of bleeding or easy bruising, a more in-depth evaluation of coagulation function including platelet assays may be indicated in consultation with pediatric hematology. Intraoperative blood product therapy should be based on the coagulation profiles. As the defect may involve platelet aggregation and function, coagulation evaluation with the ROTEM ${ }^{\circledR}$ may be particularly useful. Coagulation adjuncts such as TXA or DDAVP ${ }^{\circledR}$, both of which augment platelet function, should be considered.

\section{Data Sharing Statement}

The data supporting the findings of this case report are available from the authors.

\section{Informed Consent}

Informed consent was obtained from a parent for anesthetic care and use of patient data for publication purposes. The patient information was de-identified for publication.

\section{Author Contributions}

All authors contributed to data analysis, drafting or revising the article, have agreed on the journal to which the article will be submitted, gave final approval of the version to be published, and agree to be accountable for all aspects of the work.

\section{Funding}

There is no funding to report.

\section{Disclosure}

The authors report no conflicts of interest in this work.

\section{References}

1. Kim JM. Ketogenic diet: old treatment, new beginning. Clin Neurophysiol Pract. 2017;2:161-162. doi:10.1016/j.cnp.2017.07.001

2. Conover ZR, Talai A, Klockau KS, Ing RJ, Chatterjee D. Perioperative management of children on ketogenic dietary therapies. Anesth Analg. 2020;131:1872-1882. doi:10.1213/ANE.0000000000005018

3. Fedorovich SV, Voronina PP, Waseem TV. Ketogenic diet versus ketoacidosis: what determines the influence of ketone bodies on neurons? Neural Regen Res. 2018;13:2060-2063. doi:10.4103/1673-5374.241442

4. Kurtz I, Maher T, Hutler HN, Schambelan M, Sebastian A. Effect of diet on plasma acid-base composition in normal humans. Kidney Int. 1983;24:670-680. doi:10.1038/ki.1983.210

5. Neal EG, Chaffe H, Schwartz RH, et al. A randomized trial of classical and medium-chain triglyceride ketogenic diets in the treatment of childhood epilepsy. Epilepsia. 2009;50:1109-1117. doi:10.1111/j.1528-1167.2008.01870.x

6. Giordano C, Marchiò M, Timofeeva E, Biagini G. Neuroactive peptides as putative mediators of antiepileptic ketogenic diets. Front Neurol. 2014;5:63. doi:10.3389/fneur.2014.00063

7. Lucchi C, Marchiò M, Caramaschi E, et al. Electrographic changes accompanying recurrent seizures under ketogenic diet treatment. Pharmaceuticals. 2017;10(4):82. doi:10.3390/ph10040082

8. Valencia I, Pfeifer H, Thiele EA. General anesthesia and the ketogenic diet: clinical experience in nine patients. Epilepsia. 2002;43:525-529. doi:10.1046/j.1528-1157.2002.12501.x

9. Takeoka M, Riviello JJ, Pfeifer H, Thiele EA. Concomitant treatment with topiramate and ketogenic diet in pediatric epilepsy. Epilepsia. 2002;43:1072-1075. doi:10.1046/j.1528-1157.2002.00602.x

10. King M, Martin D, Miketic R, et al. Impact of intraoperative fluid management on electrolyte and acid-base variables during posterior spinal fusion in adolescents. Orthop Res Rev. 2020;12:69-74. doi:10.2147/ORR.S262509

11. Kraut JA, Madias NE. Metabolic acidosis: pathophysiology, diagnosis and management. Nat Rev Nephrol. 2010;6(5):274-285. doi:10.1038/ nrneph.2010.33

12. Orchard CH, Cingolani HE. Acidosis and arrhythmias in cardiac muscle. Cardiovasc Res. 1994;28(9):1312-1319. doi:10.1093/cvr/28.9.1312

13. Jacobi J. Pathophysiology of sepsis. Am J Health Syst Pharm. 2002;59(Suppl 1):S3-S8. doi:10.1093/ajhp/59.suppl_1.S3 
14. De Robertis E, Kozek-Langenecker S, Tufano R, Romano G, Piazza O, Zito Marinosci G. Coagulopathy induced by acidosis, hypothermia and hypocalcaemia in severe bleeding. Minerva Anestesiol. 2015;81:65-75.

15. Martini WZ, Holcomb JB. Acidosis and coagulopathy: the differential effects on fibrinogen synthesis and breakdown in pigs. Ann Surg. 2007;246:831-835. doi:10.1097/SLA.0b013e3180cc2e94

16. Engström M, Schött U, Romner B, Reinstrup P. Acidosis impairs the coagulation: a thromboelastographic study. J Trauma Acute Care Surg. 2006;61:624-628. doi:10.1097/01.ta.0000226739.30655.75

17. Berry-Kravis E, Booth G, Taylor A, Valentino LA. Bruising and the ketogenic diet: evidence for diet-induced changes in platelet function. Ann Neurol. 2001;49(1):98-103. doi:10.1002/1531-8249(200101)49:1<98::AID-ANA13>3.0.CO;2-2

18. Dressler A, Chiara H, Benninger F, et al. Effects of the ketogenic diet on platelet counts and global coagulation tests in childhood epilepsy. Seizure. 2020;80:31-37. doi:10.1016/j.seizure.2020.03.017

19. Vassal O, Desgranges FP, Tosetti S, et al. Risk factors for intraoperative allogeneic blood transfusion during craniotomy for brain tumor removal in children. Paediatr Anaesth. 2016;26:199-206. doi:10.1111/pan.12810

20. Bolliger D, Görlinger K, Tanaka KA, Warner DS. Pathophysiology and treatment of coagulopathy in massive hemorrhage and hemodilution. Anesthesiology. 2010;113:1205-1219. doi:10.1097/ALN.0b013e3181f22b5a

\section{Publish your work in this journal}

The International Medical Case Reports Journal is an international, peer-reviewed open-access journal publishing original case reports from all medical specialties. Previously unpublished medical posters are also accepted relating to any area of clinical or preclinical science. Submissions should not normally exceed 2,000 words or 4 published pages including figures, diagrams and references. The manuscript management system is completely online and includes a very quick and fair peer-review system, which is all easy to use. Visit http://www.dovepress.com/testimonials. php to read real quotes from published authors.

Submit your manuscript here: https://www.dovepress.com/international-medical-case-reports-journal-journal 